\title{
A Versatile Model-Based Visibility Measure for Geometric Primitives
}

\author{
Marc M. Ellenrieder ${ }^{1}$, Lars Krüger ${ }^{1}$, Dirk Stößel $^{2}$, and Marc Hanheide ${ }^{2}$ \\ 1 DaimlerChrysler AG, Research \& Technology, 89013 Ulm, Germany \\ ${ }^{2}$ Faculty of Technology, Bielefeld University, 33501 Bielefeld, Germany
}

\begin{abstract}
In this paper, we introduce a novel model-based visibility measure for geometric primitives called visibility map. It is simple to calculate, memory efficient, accurate for viewpoints outside the convex hull of the object and versatile in terms of possible applications. Several useful properties of visibility maps that show their superiority to existing visibility measures are derived. Various example applications from the automotive industry where the presented measure is used successfully conclude the paper.
\end{abstract}

\section{Introduction and Motivation}

Finding viewpoints from which certain parts of a known object are visible by a two-dimensional, perspective sensor is not a straightforward task. Especially in complex environments like industrial inspection or object recognition applications, where several geometric primitives (often called features [1]) on complex objects have to be inspected, finding an easy to calculate, versatile, storageeffective and not too simplifying visibility measure is of great importance. Up until now, several visibility measures with their particular advantages and disadvantages have been developed. However, most of them are tailor-made for specific applications so that the underlying algorithmic and conceptual framework cannot be used in other tasks. At the same time, they do not provide a consistent modeling of visibility for different feature-types like points, lines, polygons or volumetric features. Also, feature covisibility is addressed by only a few of the many existing visibility measures. Restricting the sensor positions to very few viewpoints further limits possible applications.

One of the first visibility measures for surface areas is the aspect graph by Koenderink et.al. 8]. Aspect graphs assume the object's model to be centered at the origin of a sphere which encapsulates the entire model. Every possible view of the model can then be represented as a point on that sphere. Each equivalent view on the sphere is represented by a node in the aspect graph, with the connection between graph nodes representing a possible transition between views. These transitions are due to changes in the occlusion relationships between object surfaces. However, no method whatsoever of generating the threedimensional viewpoint region of an object's aspect graph node is presented in 
this paper. In [2], Cowan and Kovesi show a way to actually generate an aspect graph, although only for simple polyhedra. They describe a method to calculate the three-dimensional region where a convex polyhedral object $\mathcal{O}$ occludes a portion of a convex polyhedral surface $\mathcal{S}$. The boundary of the occlusion zone is described by a set of separating support-planes. In the case of multiple occluding objects, the union of all non-occlusion zones is calculated. The presented algorithm has quadratic computational complexity in the number of edges of the polygon. This is of significant disadvantage in complex scenes and real applications.

Tarabanis et.al. 11 have presented a method of computing the spacial visibility regions of features. They define a feature as a polygonal and manifold subset of a single face of a polyhedral object. The visibility region of a feature is defined as the open and possibly empty set consisting of all viewpoints in free space for which the feature is visible in its entirety. Instead of calculating the visibility region directly, Tarabanis et.al. devised a three-step algorithm that calculates the occlusion region of a feature $\mathcal{T}$ in linear time (in terms of object vertices). The occlusion region is the complementary area to the visibility region with respect to free space. For each element of a subset $\mathcal{L}$ of the faces of the polyhedral object, the (polyhedral) occluding region is calculated in a similar manner to the method shown by Cowan and Kovesi [2]. The elements of $\mathcal{L}$ are those faces that satisfy certain topological and morphological properties with respect to $\mathcal{T}$. The occluding regions of all elements of $\mathcal{L}$ are merged into the complete polyhedral occlusion region $\mathcal{O}$ of the feature $\mathcal{T}$. A check for visibility of $\mathcal{T}$ from a certain viewpoint can thus be reduced to a point-in-polyhedron classification. However, since the polyhedral occlusion region $\mathcal{O}$ has to be stored as a whole, the presented method requires a considerable amount of storage memory. This makes it difficult to employ in scenarios with highly complex parts.

Another method of calculating the approximate visibility space of a feature is presented by Trucco et.al. 12. Their method restricts the possible sensor viewpoints to positions at manually fixed intervals on a spherical grid surrounding the object. The viewing direction at each grid point connects the viewpoint with the center of the spherical grid. Visibility of a feature is determined by rendering the object from each viewpoint and counting the number of pixels of the feature in the resulting image. Covisibility, i.e. the visibility of several features at once, can be determined by counting the collective number of pixels. An advantage of this approach is that it yields a quantitative and not just boolean visibility measure for each viewpoint. Also, in terms of storage memory, the approximate visibility space is very efficient. However, the restriction to a spherical grid, and the high computational complexity for the rendering process limits its use to rather simple inspection tasks.

Various other visibility measures exist in the literature. Some publications address visibility in terms of a scalar function $\mathcal{V}$ that is evaluated for each viewpoint. Khawaja et.al. 7] use the number of visible features, the number of visible mesh-faces on each feature, and the number of image pixels associated with each face as parameters of an ad-hoc postulated formula. The necessary 
parameters are generated for each viewpoint by rendering the model of the inspected object from this viewpoint. Other publications, e.g. 1], use even simpler methods: the dot-product of the viewing direction and the surface normal of the inspected feature. If the dot-product is negative, the feature is considered to be visible. Hence, visibility can only be determined correctly for strictly convex objects.

As we have seen, existing visibility measures do not account for all of the points mentioned above. Especially the lack of versatility concerning both the variety of possible applications and the correctness of the visibility determination for $1 \mathrm{~d}, 2 \mathrm{~d}$ and $3 \mathrm{~d}$ features is apparent. In the remainder of this paper, we therefore want to introduce the concept of visibility maps to determine the visibility of arbitrary geometric primitives. We will show some of their properties and demonstrate their versatility in various machine vision applications.

\section{$2 \quad$ Visibility Maps}

The term visibility map is very generic. It is used for example in computer graphics as a synonym for a graph characterizing the visible triangles of a mesh. In this paper, we use the term to describe a matrix that is used to determine the visibility of a geometric primitive. In principle, a visibility map is defined for points on the surface of an object. It is calculated by projecting the inspected object (and possibly the whole scene) onto a unit sphere centered at the point on the object for which the map is calculated. The unit sphere is then sampled at constant azimuth / elevation intervals $\nu$ and the boolean information whether something has been projected on the current point on the sphere or not, is transcribed into a matrix called visibility map. Fig. 11 illustrates this concept.

\subsection{Calculating Visibility Maps}

Calculating visibility maps can be implemented effectively, if the object surface geometry is given as a triangulated mesh with three-dimensional vertex coordinates $v_{i}$. The vertices are projected onto a unit sphere centered at the point whose visibility has to be calculated. Without loss of generality, we assume this point to be the origin of the coordinate system. Using the four-quadrant arcustangent function the vertices' spherical coordinates $\theta$ (azimuth), $\phi$ (elevation) and $r$ (radius) result to

$$
\begin{aligned}
& \theta\left(\tilde{v}_{i}\right)=\arctan 2\left(v_{i, y}, v_{i, x}\right), \\
& \phi\left(\tilde{v}_{i}\right)=\frac{\pi}{2}-\arctan 2\left(v_{i, z}, \sqrt{v_{i, x}^{2}+v_{i, y}^{2}}\right), \text { and } \\
& r\left(\tilde{v}_{i}\right) \equiv 1 .
\end{aligned}
$$

Suppose, two vertices $v_{i}$ and $v_{j}$ are connected by a mesh-edge. Then, the meshedge is sampled at $k$ intervals. The sampled edge points are then projected onto 
the sphere and an approximate spherical mesh-triangle is constructed by connecting the projected edge samples using BRESENHAM's algorithm. The resulting triangle-outline is filled using a standard flood-fill algorithm. This process is repeated for every triangle of the object. Afterwards, the unit sphere is sampled in both azimuth and elevation direction at intervals $\nu$ and the result whether something has been projected or not is transcribed into the matrix $M$, i.e. the visibility map. To account for numerical errors and to get a smooth visibility map we further apply standard dilation / erosion operators to $M$. The computational complexity of this method is $\mathcal{O}(n)$ for objects comprised of $n$ triangles. An example visibility map can be seen in Fig. 1.

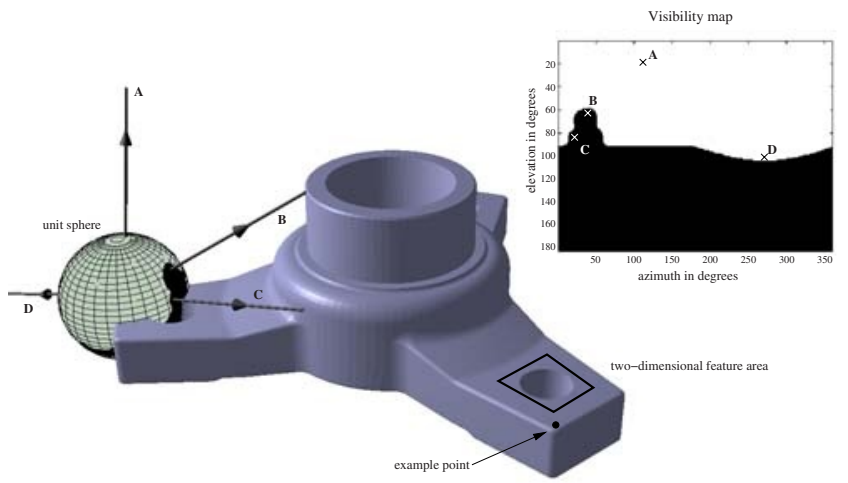

Fig. 1. Visibility map of a point on a flange. The same point on the right arm of the flange is highlighted. Black regions in the visibility map represent viewing directions, where a camera positioned at the center of the sphere would see parts of the flange. The position of four example viewing directions (A-D) are marked in the map. For illustration purposes, the size of the unit sphere has been exaggerated

\subsection{Distance Transform}

Sometimes, it can be useful to quickly determine viewing directions where the visibility of features is as stable as possible towards slight viewpoint deviations. This means, the sensors should be positioned as far away as possible from the occlusion-zone boundaries. One way to achieve this is to calculate the distance transform of a visibility map. However, since visibility maps are spherical projections, we need to use a spherical rather than an Euclidean distance measure. Using the Haversine function $h(x)=\sin ^{2}(x / 2)$, the distance of two points $p_{1}=\left(\theta_{1}, \phi_{1}\right)$ and $p_{2}=\left(\theta_{2}, \phi_{2}\right)$ on the unit sphere can be expressed as

$$
d\left(p_{1}, p_{2}\right)=2 \cdot \arctan 2(\sqrt{a}, \sqrt{1-a}),
$$

where $a=h\left(\theta_{2}-\theta_{1}\right)+\cos \left(\theta_{1}\right) \cos \left(\theta_{2}\right) h\left(\phi_{2}-\phi_{1}\right)$. By convention, we define $d<0$ for visible viewing directions and $d>0$ for occluded directions. The actual distance transformation is calculated using standard propagation algorithms [3] which efficiently exploit the transitivity of the minimum relation. 

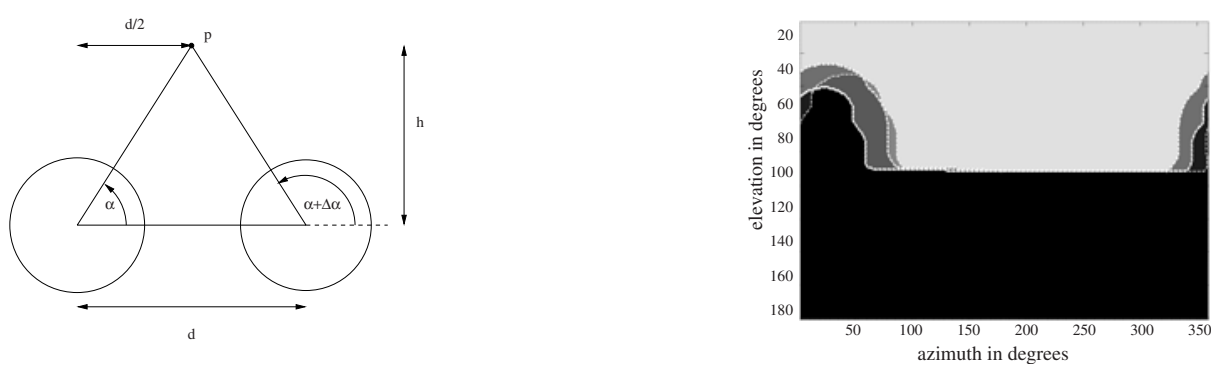

Fig. 2. Geometric relations for $2 \mathrm{~d}$ feature visibility (left) and full feature visibility map (5) of the polygonal area shown in Fig. 1. The different corner point visibility maps are shown for illustration purposes

\subsection{Visibility of $2 \mathrm{D}$ and $3 \mathrm{D}$ Features}

In many applications a visibility measure for $2 \mathrm{D}$ or even $3 \mathrm{D}$ features is required. By concept however, the visibility map is defined for one single point on the surface of an object. Nevertheless, it can be generalized for higher-dimensional geometric primitives, i.e. lines, polygonal areas, and volumes, if it is assumed that the camera is far away at distance $h$ in comparison to the maximum extension $d$ (i.e. the length of the longest eigenvector) of the primitive. This is equal to the assumption of using the same camera direction (azimuth / elevation) for each of the $N$ corner points of the primitive. The resulting error is depending on the relation $d / h$ and can be estimated as follows: let $\boldsymbol{p}$ be a $3 \mathrm{D}$-point that is projected onto two unit spheres at located at a distance $d$. Figure 2 shows the geometric relations in the plane spanned by the sphere centers and $\boldsymbol{p}$. The point is projected onto the first sphere at an elevation angle $\alpha$ and at elevation $\alpha+\Delta \alpha$ onto the second. It is clear, that for any fixed $h, \Delta \alpha \rightarrow \max$ if $\boldsymbol{p}$ is located in the middle of the spheres at $d / 2$. We have

$$
\Delta \alpha=\arctan \left(\frac{-2 h}{d}\right)-\arctan \left(\frac{2 h}{d}\right) .
$$

In order to get a value of $\Delta \alpha$ that is smaller than the angular spacing $\nu$ of the visibility map, we need to find $\left|\Delta \alpha\left(\frac{d}{h}\right)\right|<\nu$. From (3) we get

$$
\frac{d}{h} \leq-\frac{1}{2} \tan \frac{\nu}{2}
$$

For a typical sampling interval of $\nu=1$, this results to $d / h \leq 0.004$.

For higher dimensional primitives, there are basically two notions of visibility: full visibility and partial visibility. In industrial inspection applications, full visibility of a feature is often required, e.g. to check for complete visibility of bar codes. In other applications, e.g. object recognition applications, partial visibility might however be sufficient. For primitives, where full visibility is required, it can be calculated by forming a union of the visibility maps of each corner point, leading to full feature visibility maps 


$$
M=\bigcup_{k=0}^{N-1} M_{k, \text { corner }}
$$

This concept is applicable for 2D polygonal areas, e.g. single meshes, or parts of $3 \mathrm{D}$ primitives, e.g. several sides of a cube. It can be further extended to the covisibility of several features, resulting in the combined feature visibility map $\bar{M}$. If full visibility is not required or possible, e.g. in case of three-dimensional primitives, (5) will not yield a correct visibility measure. Nevertheless, the visibility map can also be used, if the visibility maps of the primitives vertices $M_{k \text {,corner }}$ are combined into partial feature visibility maps by

$$
M=\sum_{k=0}^{N-1} 2^{k-1} M_{k, \text { corner }}
$$

Then, the visibility of each vertex can be evaluated separately. Using the distance transform of the visibility maps of two vertices $\boldsymbol{p}_{1}$ and $\boldsymbol{p}_{2}(2)$ it is also possible to calculate the visible length $d_{\mathrm{vis}}$ of a mesh-edge connecting these vertices. Figure 3 shows the geometric relations in the plane $E$ spanned by $\boldsymbol{p}_{1}, \boldsymbol{p}_{2}$ and viewpoint $\boldsymbol{v}$. The plane cuts a great circle from both visibility maps. All angles and distances used in the following are measured in this plane. For the sake of simplicity, we define a coordinate system with unit vectors $\boldsymbol{x}_{\mathrm{E}}$ and $\boldsymbol{y}_{\mathrm{E}}$ originating in $\boldsymbol{p}_{1}$. Then, the $x_{\mathrm{E}}-y_{\mathrm{E}}$ components of $\boldsymbol{k}$ are given by

$$
k_{x_{\mathrm{E}}}=\left(1-\frac{\tan \left(\alpha_{1}+\delta_{1}\right)}{\tan \left(\alpha_{2}+\delta_{2}\right)}\right)^{-1} \cdot d, k_{y_{\mathrm{E}}}=d \cdot \tan \left(\alpha_{1}+\delta_{1}\right) .
$$

Angles $\alpha_{k}$ and $\delta_{k}$ can be directly drawn from the visibility maps and their distance transforms. Calculating the intersection of $g_{3}$ and the $x_{\mathrm{E}}$-axis results in

$$
d_{\mathrm{vis}}(d)=\left(v_{x_{\mathrm{E}}}-v_{y_{\mathrm{E}}}\right) \frac{v_{x_{\mathrm{E}}}-k_{x_{\mathrm{E}}}}{v_{y_{\mathrm{E}}}-k_{y_{\mathrm{E}}}} .
$$

Here, $v_{x_{\mathrm{E}}}$ and $v_{y_{\mathrm{E}}}$ describe the coordinates of the viewpoint projected onto the plane $E$. If applied to each mesh-edge, (8) allows to calculate the visible area or volume of the primitive directly from the visibility maps (Fig. 3). In viewpoint planning applications this property can be used to directly assign a quantitative visibility value to a viewpoint.

\subsection{Visibility Ratio and Memory Efficiency}

By using visibility maps, it is possible to estimate the size of the space, from which one or more features are completely visible. This can be used to determine, whether e.g. a sensor head mounted on a robotic arm can be positioned such that certain primitives are visible. For this, we define the term visibility ratio of a single feature $F_{j}$ :

$$
V\left(F_{j}\right)=\frac{\text { visible area of } M\left(F_{j}\right)}{\text { total area of } M\left(F_{j}\right)} .
$$



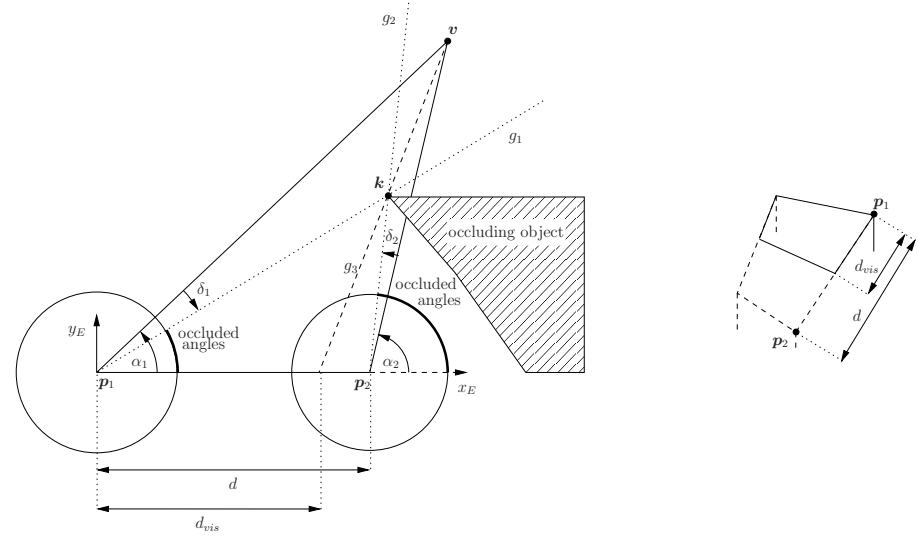

Fig. 3. The geometric relations in the plane spanned by $\boldsymbol{p}_{1}, \boldsymbol{p}_{2}$ and viewpoint $\boldsymbol{v}$ (left) for calculating the visible area of a partially visible mesh facet (right). All units are measured in this plane. Angles $\delta_{1,2}$ are derived from the visibility maps' distance transforms (2)

This property can be interpreted as the relation of the visible solid angle of the the feature to the full sphere. Equally, we define the combined feature visibility ratio $\bar{V}\left(C_{i}\right)$ of the features associated to camera $i$ by using the combined feature visibility map $\bar{M}\left(C_{i}\right)$. It has to be noted that for full feature visibility maps $\bar{V}<V \leq 0.5$ for all two-dimensional primitives other than a single line, since they are on the surface of the inspected objects and are thus not visible from viewpoints on the backside of the object. One can therefore assume that there is at least one large connected region in the visibility map. Hence, visibility maps can be stored very effectively by using simple run-length-encoding as a means to compress them.

\section{Applications Using Visibility Maps}

To show the versatility of the visibility map, we are going to present several systems using the presented concepts in various applications from object recognition to viewpoint planning. In our opinion, this versatility together with the simplicity of its concept renders the visibility map superior to other existing visibility measures.

\subsection{Object Recognition and Pose Estimation}

One of the first applications, where visibility maps have been used, was object recognition. In [9] a system for multi-feature, multi-sensor classification and localization of $3 \mathrm{D}$ objects in $2 \mathrm{D}$ image sequences is presented. It uses a hypothesizeand-test approach to estimate type and pose of objects. Characteristic Localized Features (CLFs), e.g. contours, 3D corners, etc., are extracted from the geomet- 

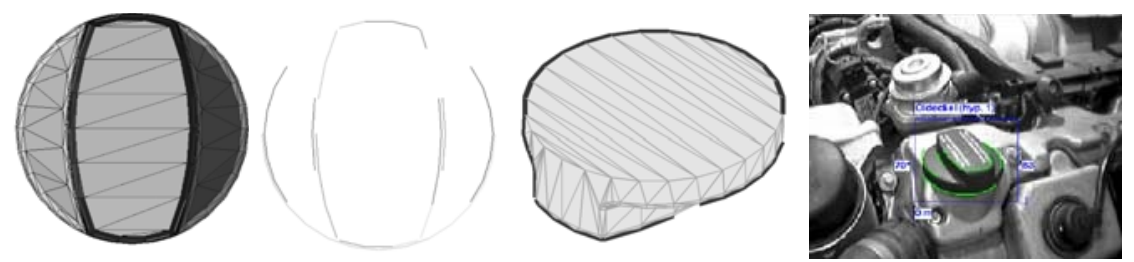

Fig. 4. From left to right: An oil cap, its CLF graph seen from two different viewpoints, aligned to the image. Visibility of the CLFs was calculated using visibility maps

ric models of the different objects and viewpoint dependant graphs of the CLFs projected onto the image plane are generated for each pose and object type hypothesis. By using elastic graph matching algorithms [6], the graph is aligned with the features in the image. Viewpoint dependant graph rendering is only possible, since visibility of each CLF was calculated using the visibility map. The system is used for both optical inspection for quality control and airport ground-traffic surveillance. An example CLF-graph and the recognized object type and pose is shown in Fig. 4. Since there are typically several hundreds of CLFs per object whose visibility has to be evaluated several times, both storage memory and speed of the employed visibility measure are crucial.

\subsection{Optimal Sensor-Feature Association}

The cost of automatic inspection systems for quality control directly depends on the number of installed sensors. Clearly, more than $r$ sensors for $r$ features is therefore not an option, less, i.e. $k<r$, sensors would even be better. For $r$ feature areas, there are $2^{r}-1$ possible feature combinations that can be assigned to one sensor. To find the optimal association, we need to define a criterion that compares different combinations. The combined feature visibility map's visibility ratio can be used for finding an optimal assignment matrix $C$ of size $k \times r$ with $k \leq r$ whose column-sums equal to 1 and whose elements $C_{i j}$ are 1 , if feature $j$ is associated to camera $i$. Using a row vector $C_{i}$ that represents the associated features of camera $i$, the weighted visibility ratio $\tilde{V}$ of an assignment matrix $C$ with $k$ rows

$$
\tilde{V}(C)=\frac{1}{k} \sum_{i=1}^{k} \bar{V}\left(C_{i}\right)
$$

to compare all possible assignments is introduced in [4. Based on this equation, an algorithm to find the optimal sensor-feature association matrix $C_{\text {opt }}$ with a minimum number of sensors is also presented.

\subsection{Entropy Based Viewpoint Selection}

Stel et al. 10] extend the notion of CLFs to an entropy based viewpoint selection method. There, the best viewpoints for distinguishing different aggregate models, i.e. different bolt-nut combinations (Fig. 5), and their respective 2D 

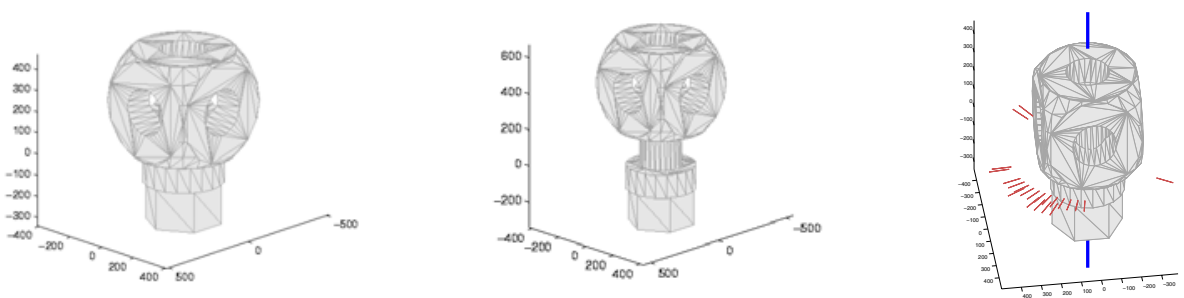

Fig. 5. Two different nut-bolt aggregations (left / middle). The entropy index mapped into visibility maps according to [10] determines the 20 best (light lines) and worst viewing directions (bold lines from above / beneath) to distinguish them (right)
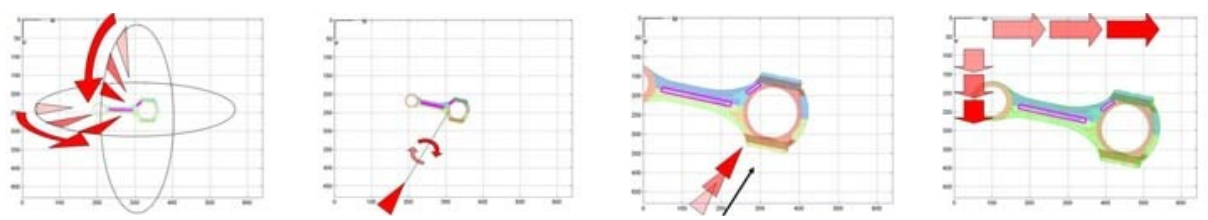

Fig. 6. Simple four step algorithm to find a good initial viewpoint using visibility maps

projections are calculated. The collective entropy mapped onto a visibility map is used to derive a distinction measure for different viewing directions. High entropy determines bad viewing directions, low entropy values mark good ones.

\subsection{Optimal Camera and Illumination Planning}

Another application using visibility maps is presented in 4. There, the optimal viewpoints for industrial inspection tasks are calculated from a geometric model of the inspected objects (Fig. 6). The distance transform of the visibility map allows to automatically position the sensor in a viewing direction that is far from vanishing points in the viewing volume. Further refinements regarding additional viewpoint requirements, e.g. minimum resolution or viewing angle, are implemented using convex scalar functions dependant on the viewpoint. Using the distance transformation of the feature visibility maps, a scalar cost can be assigned to each six-dimensional viewpoint . The final, optimal viewpoints are found by minimizing the scalar cost functions. Similarly, the visibility map can be used to find an illumination position [5], from which a desired feature illumination condition, e.g. specular or diffuse reflection, can be observed.

\section{Summary and Conclusion}

We have presented a versatile model-based visibility measure for geometric primitives called visibility map. It is easy to calculate, memory efficient, quick to use, and provides an accurate way of determining the visibility of $1 \mathrm{~d}, 2 \mathrm{~d}$, or $3 \mathrm{~d}$ geometric primitives of triangulated meshes from viewpoints outside the convex hull 
of the whole object. Several applications that prove the versatility and usefulness of this concept for object recognition, pose estimation, as well as sensor and illumination planning are presented. Since a visibility map has to be calculated only once, a test for visibility from a specific viewing direction comprises only a table lookup. In our opinion, this versatility together with the simplicity of its concept renders the visibility map superior to other existing visibility measures. One shortcoming of the visibility map, however, is the fact that visibility can only be determined correctly for viewpoints outside of the complex hull of the object.

\section{References}

1. S. Y. Chen and Y. F. Li, A method of automatic sensor placement for robot vision in inspection tasks, in Proc. IEEE Int. Conf. Rob. \& Automat., Washington, DC, May 2002, pp. 2545-2550.

2. C. Cowan and P. Kovesi, Automatic sensor placement from vision task requirements, IEEE Trans. Pattern Anal. Machine Intell., 10 (1988), pp. 407-416.

3. O. Cuisenaire, Distance transformations: fast algorithms and applications to medical image processing, PhD thesis, Univ. cath. de Louvain, Belgium, Oct. 1999

4. M. M. Ellenrieder and H. Komoto, Model-based automatic calculation and evaluation of camera position for industrial machine vision, in Proc. SPIE Computational Imaging III. 2005.

5. M. M. Ellenrieder et al., Reflectivity Function based Illumination and Sensor Planning for industrial inspection, Proc. SPIE Opt. Metrology Symp., Munich, 2005

6. E. Kefalea, O. Rehse, and C. v. d. Malsburg, Object Classification based on Contours with Elastic Graph Matching, Proc. 3rd Int. Workshop Vis. Form, 1997

7. K. Khawaja et al. , Camera and light placement for automated visual assembly inspection, in Proc. IEEE Int. Conf. Robotics 83 Automation, Minneapolis, MN, April 1996, pp. 3246-3252.

8. J. J. Koenderink and A. J. van Doorn, The internal representation of solid shape with respect to vision, Biol. Cybern., 32 (1979), pp. 151-158.

9. T. Klzow and M. M. Ellenrieder, A general approach for multi-feature, multi-sensor classification and localization of $3 \mathrm{~d}$ objects in $2 \mathrm{~d}$ image sequences, in Proc. SPIE Electronic Imaging Conf., vol. 5014, 2003, pp. 99-110.

10. D. Stel et al., Viewpoint selection for industrial car assembly, in Springer LNCS., vol. 3175 - Proc. $26^{\text {th }}$ DAGM Symp. 2004, pp. 528-535.

11. K. A. Tarabanis et al., Computing occlusion-free viewpoints, IEEE Trans. Pattern Anal. Machine Intell., 18 (1996), pp. 279-292.

12. E. Trucco et al., Model-based planning of optimal sensor placements for inspection, IEEE Trans. Robot. Automat., 13 (1997), pp. 182-194. 